\title{
Philosophiques
}

\section{La pensée évolutionniste dans la théorie économique néoclassique}

\section{Alan Kirman}

Volume 25, numéro 2, automne 1998

Les modèles d'évolution en économie et en sciences sociales

URI : https://id.erudit.org/iderudit/027488ar

DOI : https://doi.org/10.7202/027488ar

Aller au sommaire du numéro

\section{Éditeur(s)}

Société de philosophie du Québec

ISSN

0316-2923 (imprimé)

1492-1391 (numérique)

Découvrir la revue

Citer cet article

Kirman, A. (1998). La pensée évolutionniste dans la théorie économique néoclassique. Philosophiques, 25(2), 219-237. https://doi.org/10.7202/027488ar
Résumé de l'article

Cette communication traite de l'utilisation des concepts évolutionnaires en économie. Une approche a été d'utiliser Vidée de révolution comme une vague analogie. Il y a deux exemples de ce type d'approche. Certains; comme Friedman, ont utilisé la notion de sélection naturelle afin de justifier le modèle standard de la théorie économique, celui d'Arrow-Debreu. D'autres ont utilisé l'idée d'évolution comme base à une critique de la nature fermée et statique de ce modèle. Une autre approche a été de prendre l'idée de l'évolution au sérieux, comme cela a été fait en théorie des jeux évolutionnaires. Je suggère que même dans ce cas, le modèle standard est incompatible avec un point de vue évolutionniste et qu'il ne suffit pas d'utiliser l'évolution comme outil pour sélectionner parmi les équilibres statiques. Des modèles économiques compatibles avec une approche évolutionnaire doivent nécessairement être très différents des modèles standards qu 'on utilise aujourd'hui. 


\title{
LA PENSÉE ÉVOLUTIONNISTE DANS IA THÉORIE ÉCONOMIQUE NÉOCLASSIQUE
}

\author{
PAR \\ ALAN KIRMAN
}

\begin{abstract}
RESUMÉ : Cette communication traite de l'utilisation des concepts évolutionnaires en économie. Une approche a été d'utiliser l'idée de l'évolution comme une vague analogie. Il y a deux exemples de ce type d'approche. Certains, comme Friedman, ont utilisé la notion de sélection naturelle afin de justifier le modèle standard de la théorie. économique, celui d'Arrow-Debreu. D'autres ont utilisé l'idée d'évolution comme base à une critique de la nature fermée et statique de ce modèle. Une autre approche a été de prendre l'idée de l'évolution au sérieux, comme cela a été fait en théorie des jeux évolutionnaires. Je suggère que même dans ce cas, le modèle standard est incompatible avec un point de oue évolutionniste et qu'ul ne suffit pas d'utiliser l'évolution comme outil pour sélectionner parmi les équilibres statiques. Des modèles économiques compatibles avec une approche évolutionnaire doivent nécessairement être très différents des modèles standards qu'on utilise aujourd'hui.
\end{abstract}

ABSTRACT: This article discusses the use of evolutionary concepts in economic theory. One approach has been to use evolution as a loose analogy: There have been two principle examples of this approach. Those such as Friedman who have used the notion of "natural selection "to justify the standard model in economic theory, that of Arrow-Debreu and those who have used it to criticize that model for its closed and static nature. A second approach has been to take the idea of evolution more seriously as has been done in evolutionary game theory. I suggest that even in this latter case the standard model is incompatible with a truly evolutionist view and it is not enough to use evolution as a tool to select amongst static equilibria. Economic models which are consistent with an evolutionary approach have necessarily to be very different from the standard ones in use.

L'utilisation du terme évolutionniste en économie a une longue tradition. Marshall a été influencé par des travaux de biologistes. Il faisait fréquemment allusion à des termes biologiques et utilisait en particulier le terme de sélection naturelle. Il est peut-être encore plus frappant que Darwin lui-même ait reconnu sa dette envers Malthus dans le développement de sa propre théorie de l'évolution. On peut 
adopter deux points de vue très différents au sujet de l'utilisation en économie de termes tels que évolution. Le premier consiste à considérer simplement l'utilisation de termes biologiques comme un moyen de se référer à une analogie générale. Cette approche constitue à la fois un outil servant à justifier la théorie standard de l'économie et une façon de critiquer ceux que l'on pourrait décrire de diverses façons comme les économistes néoclassiques, les économistes du courant dominant, les économistes orthodoxes ou même les économistes walrasiens.

Ceux qui cherchent à fournir une justification à la notion standard d'équilibre ont l'idée de donner une explication de l'évolution d'une économie vers un équilibre. Je suggère que la structure même du modèle de base de l'économie, celui d'Arrow et Debreu, empêche une utilisation autre que très superficielle de l'approche évolutionniste.

Ceux qui critiquent l'économie standard en utilisant l'analogie avec l'évolution biologique expliquent tout simplement que l'on doit faire une distinction entre un système fermé, dans lequel les individus ont des caractéristiques fixes, et un système qui est en quelque sorte a illimité » et en expansion. Dans cette optique, la notion même d'équilibre n'a pas de sens. Mais l'utilisation du terme évolution est très souvent superficielle.

Le deuxième point de vue est de prendre au sérieux le paradigme évolutionniste, de spécifier ses caractéristiques et de raisonner alors en termes de reproduction et de sélection. Dans cet article, étant donné la difficulté de réconcilier l'analogie biologique générale avec le modèle de base de la théorie de l'équilibre, j'expliquerai comment la première a été utilisée dans d'autres approches formelles de la théorie économique. Pour illustrer ceci, je parlerai des travaux récents qui ont utilisé l'évolution et l'adaptation pour sélectionner parmi les équilibres de modèles bien formulés, mais statiques. C'est en particulier l'approche qui a été adoptée en théorie des jeux.

Cependant, mon projet ici est de suggérer que nous sommes prêts à aller plus loin tout en prenant au sérieux l'approche évolutionniste. À cette fin, il faut considérer l'économie comme un système complexe en continuel développement. Mais même en procédant ainsi, nous ne pourrons pas coller complètement au contexte biologique. Plus important encore, si l'analogie biologique peut véritablement avoir un sens en économie, la structure générale des modèles économiques correspondants doit être très différente de celle des modèles standards avec lesquels nous travaillons d'habitude. 
La parution récente de deux livres à ce sujet ${ }^{1}$ atteste de l'intérêt croissant dont bénéficie cette approche et justifie que je jette un coup d'œil aux développements de ce domaine.

Cependant, mon projet ici est de suggérer que, si nous sommes prêts à prendre au sérieux l'approche évolutionniste en considérant l'économie comme un système complexe en développement, nous ne pourrons pas coller complètement au contexte biologique et qu'en outre, même dans les modèles où l'analogie biologique a un sens, la structure générale devra être relativement différente de celle des modèles standards avec lesquels nous travaillons.

Bien que d'autres auteurs avant lui aient fait référence à l'économie comme système biologique, c'est probablement à partir de Marshall que l'on peut regarder l'utilisation qui a été faite en économie de l'analogie évolutionniste. De façon caractéristique, les économistes ne font pas de distinction entre évolutionniste et darwinien. Cependant, il semblerait que Marshall ait été plus influencé par Herbert Spencer ${ }^{2}$ que par Darwin lui-même. En effet, Hodgson va même jusqu'à dire que :

Personne ne peut entièrement comprendre ou interpréter Marshall sans avoir lu les travaux fondamentaux de Spencer, comme ses Premiers Principes. Non seulement Marshall (1890) a lui-même fait référence plus souvent à Spencer, mais aussi pendant la période cruciale 1880-1900 Spencer avait une influence beaucoup plus importante que Darwin dans la pensée occidentale, (Bowler) (1983) ${ }^{3}$.

Jusqu ici, la plupart des économistes intéressés par l'utilisation de l'approche évolutionniste se réfèrent systématiquement à Darwin. Ce n'est pas totalement sans importance puisque Spencer utilisait délibérément le terme de persistance du plus apte alors que Darwin était moins disposé à faire référence à l'optimisation. Il vaut donc la peine d'éclaircir ce que l'on entend par approche darwinienne. Une formulation générale en est fournie par Lewontin ${ }^{4}$ qui identifie les quatre principes de base de l'approche darwinienne comme :

1. Le principe de variation : il doit y avoir des variations entre les membres d'une espèce ou d'une population; ces variations doivent être aveugles, aléatoires ou non intentionnelles ;

2. Le principe d'hérédité : la progéniture doit ressembler davantage à ses parents qu'aux autres membres de son espèce ;

3. Le principe de sélection naturelle : les organismes les mieux adaptés laissent une progéniture de plus en plus nombreuse, soit immédiatement, soit dans le futur ;

1. Voir J. Weibull, Evolutionary Game Theory, Cambridge, MIT Press, 1996 et L. Samuelson, Evolutionary Games and Equilibrium Selection, Cambridge, MIT Press, 1997.

2. H. Spencer, First Principles, $5^{\mathrm{e}}$ édition, Londres, Williams and Norgate, 1890.

3. G. M. Hodgson, Economics and Evolution: Bringing Life Back into Economics, Cambridge/Ann Arbor, Polity Press/University of Michigan Press, 1993.

4. R. Lewontin, « Adaptation ", Scientific American, 239, 1978, p. 212-230. 
4. Le principe de lutte pour la vie: les variations ou combinaisons de gènes qui sont préservées sont celles qui accordent un avantage dans la lutte pour la survie.

Nelson et Winter ${ }^{5}$ donnent deux raisons pour lesquelles il y a un intérêt à rechercher un paradigme économique basé sur ces principes. Premièrement, on peut prendre en compte le changement de façon explicite et, deuxièmement, on peut se débarrasser de la dépendance à l'égard de l'optimisation au niveau individuel. Si c'est en effet la motivation nécessaire à l'adoption d'une approche plus biologique, il suffit d'examiner le développement de la théorie économique pour voir pourquoi son modèle de base, celui d'Arrow et Debreu, est incompatible avec ces objectifs. Je pense qu'il suffit d'examiner l'évolution du modèle standard pour comprendre pourquoi il en serait ainsi.

C'est presque devenu maintenant un lieu commun de dire que l'économie formalisée s'est elle-même modelée autour de la mécanique classique. Pendant longtemps, ce processus a été considéré comme presque subconscient. Cependant, Ingrao et Israel ${ }^{6}$ expliquent bien clairement, et en détail, cette relation et soulignent l'adoption consciente d'un certain nombre de concepts issus de la physique. Mirowski va plus loin et dit :

L'école dominante de la théorie économique occidentale, que nous appellerons l'économie néoclassique, a souvent été dans le passé glorifiée et condamnée pour avoir été érigée à partir de la physique standard. Cependant, à mon grand étonmement, je réalise que personne n'a jamais sérieusement examiné les parallèles historiques. Quand on s'en donne la peine, on découvre rapidement que les ressemblances entre les théories sont étranges, et une raison pour laquelle elles sont étranges est que les pères fondateurs de la théorie économique néoclassique ont hardiment copié les théories de la physique régnant dans les années 1870 . Plus on creuse et plus on réalise que ces néoclassiques n'ont pas imité la physique de façon décousue ou superficielle; non, ils ont copié leurs modèles le plus souvent terme après terme et symbole après symbole, et ils l'ont dit.

Donc, selon Mirowski et beaucoup d'autres, la métaphore physique a triomphé en économie, au moins jusqu'à très récemment. Une telle vision des choses, ainsi que l'évidente dépendance de l'économie vis-à-vis des notions d'optimisation et d'équilibre sont complètement compatibles avec le fait que la métaphore biologique en économie disparaît virtuellement entre la mort de Marshall et 1970. Hodgson ${ }^{7}$ trouve sept travaux en économie entre 1914 et 1970 dont le titre comporte les mots évolution ou évolutionniste. Comme le remarque Shove dans son article écrit à l'occasion du centenaire de la mort de Marshall : « Il y eu un retour de l'approche mécanique par

5. R. Nelson et S. Winter, An Evolutionary Theory of Economic Change, Cambridge, Harvard University Press, 1982.

6. B. Ingrao et G. Israel, The Invisible Hand, Cambridge, MrT Press, 1990.

7. G. M. Hodgson, Economics and Evolution: Bringing Life Back into Economics. 
opposition à l'approche biologique dans l'économie dominante après la mort de Marshall. Pour citer Hodgson : "De 1914 à 1970 l'idée même d'une "biologie économique" était - à des exceptions périphériques près - morte ${ }^{8}$.

Après cela, on pourrait argumenter en disant que divers auteurs, frustrés par le cadre rigide qu'imposent les variantes du modèle de Arrow-Debreu, ont amorcé un retour à une vision biologique. Il semble donc que la dissociation de l'économie, ou au moins de l'économie dominante, de la tradition évolutionniste s'explique de façon limpide. Le lecteur aura noté que j’ai exclu Schumpeter et Hayek de cette discussion, et pourra interpréter ceci en considérant quils nétaient pas dominants ou, plus vraisemblablement, quils n'ont pas fait d'utilisation littérale de la métaphore biologique. Il vaut la peine de noter, comme Nelson et Winter ${ }^{9}$, que Schumpeter avait des mots très durs pour parler de la perte d'usage de la métaphore évolutionniste en économie.

Une explication claire et nette du développement de la théorie économique durant ce siècle pourrait être alors que cette théorie était essentiellement dominée par les méthodes analytiques de la mécanique classique et que la physique triomphait comme modèle pour l'économie. Ceci s'est fait contre la biologie, qui met l'accent sur l'adaptation au détriment de l'optimisation. C'est malheureusement une vision beaucoup trop simple de ce qui s'est passé à la fois en économie et en biologie. D'abord, Hayek a utilisé systématiquement les arguments évolutionnistes pour justifier l'émergence de l'ordre social. Bien qu'on ne le considère pas, et de loin, comme un économiste néoclassique dans le sens habituel du terme, il est curieux qu'il ait maintenu que l'émergence du système de marché soit un résultat des forces évolutionnistes agissant non seulement sur le comportement des individus, mais aussi sur les règles et.la structure sociale. Sa vision du résultat comme étant optimal serait, bien sûr, contestable d'un point de vue strictement évolutionniste. Depuis, dans le courant dominant, on a justifié à maintes reprises en économie tout comportement optimisateur comme le résultat d'un comportement adaptatif ou de sélection naturelle. Les remarques de Friedman ${ }^{10}$ à ce sujet sont si célèbres qu'il est inutile de les répéler ici. Les objections logiques à sa position sont claires, et elles reflètent une opinion largement répandue. Alchian a développé ce raisonnement et donné l'argument selon lequel la sélection naturelle engendrerait un comportement optimisateur. Si tel est le cas, de nombreux économistes ont justifié l'idée que l'on pourrait tout aussi bien supposer que la rationalité est complète et étudier les équilibres qui émergeraient. Ainsi, Harry Johnson affirme sans réserve :

8. G. M. Hodgson, Economics and Evolution: Bringing Life Back into Economics.

9. R. Nelson et S. Winter, An Evolutionary Theory of Economic Change.

10. M. Friedman, "The Methodology of Positive Economics " dans Essays in Positive Economics, Chicago, University of Chicago Press, 1953, p. 3-43. 
On a montré... que si les firmes cherchent consciemment ou pas à maximiser les profits et minimiser les coûts, la concurrence éliminera les firmes inefficaces ; et ceci que le comportement des consommateurs soit rationnel ou purement aléatoire, les courbes de demande pour un produit tendront à décroître comme clans l'analyse Marshallienne. En conséquence, il est possible pour les économistes de traiter l'économie comme un système interdépendant répondant aux changements selon certains principes généraux de type rationnel, avec consicérablement plus de confiance que cela semblait justifié trente ans plus tôt $\mathrm{t}^{\mathrm{t}}$.

C'est la position récemment adoptée de façon encore plus explicite par Lucas quand il dit :

En général nous concevons ou modélisons un individu comme une collection de règles de décision (règles qui dictent l'action à prendre dans les situations données) et un ensemble de préférences utilisé pour évaluer les résultats qui émergent de combinaisons particulières de situation-action. Ces règles de décision sont constamment revues et révisées : de nouvelles décisions sont essayées et testées par rapport à l'expérience et les règles qui produisent des résultats désirés supplantent celles qui ne le font pas. J'utilise le terme " adaptatif " pour faire référence à ce processus de tâtonnement qui constitue nos modèles de comportement ${ }^{\mathrm{L}}$.

Cependant, Lucas poursuit en disant que nous pouvons sans risque ignorer les dynamiques de ce processus puisque :

Techniquement, je considère l'économie comme l'étude des règles de décision qui constituent les états stationnaires d'un processus adaptatif, règles de décision que l'on trouve opérantes dans une série de situations et ne sont donc plus révisées de façon appréciable quand s'ajoute une expérience supplémentaire ${ }^{13}$.

Donc, la différence entre l'approche de nombreux économistes qui se fient à la théorie standard et ceux qui argumentent en faveur d'un style d'analyse biologique concerne la stabilité de l'ajustement vers l'équilibre et la vitesse de cet ajustement. Le principe de base de ceux qui se concentrent sur l'équilibre est que les individus d'une économie apprennent ou sont sélectionnés relativement rapidement pour se comporter de façon optimale et que l'environnement économique change suffisamment lentement de sorte que, dans la situation résultante, ils n'ont pas besoin de continuer à apprendre. Ceci est en opposition avec l'affirmation d'autres auteurs qui, comme moi, considèrent que l'économie est un système adaptatif complexe, et que le processus même d'apprentissage et d'adaptation, et la réaction aux conséquences de cette adaptation engendrent des dynamiques hautement compliquées qui peuvent très bien ne pas converger vers un équilibre économique standard. Cependant, il est clair que les concepts d'adaptation et de sélection ne sont pas bannis

11. H. Johnson, « The Economic Approach to Social Questions ", Economica, vol. 35, 1968, p. 5.

12. R. Lucas, "Adaptive Behaviour and Economic Theory", Journal of Business, vol. 59, 1988, p. S401-S426.

13. Ibid. 
de la théorie économique, sauf dans les modèles d'équilibre général les plus rigoureux. Ils ont été utilisés pour justifier l'hypothèse d'une optimisation complexe par des agents économiques, plutôt que comme une solution de rechange à cette optimisation.

Il est vrai aussi qu'une telle approche n'est pas restée incontestée, même à ses débuts. Winter a engagé une controverse avec Friedman :

Il y a alors une difficulté fondamentale dans les formulations existantes de l'argument de sélection, une difficulté qui est enracinée dans le fait que les déviations relatives par rapport à la maximisation du profit de firmes différentes peuvent changer au cours du temps. Puisqu'il n'y a pas eu de traitement attentif du processus dynamique par lequel certains modèles de comportement sont considérés conme viables et d'autres non, on a oublié de noter que les firmes ne peuvent pas en général être rangées de façon non ambiguë du point de vue de leur proximité à un comportement maximisateur. Un tel classement doit, en général, présumer un état particulier de l'environnement, mais l'enviromnement est modifié par le processus dynamique lui-même ${ }^{1 / 4}$.

Dans le même temps, la biologie n'a pas été exempte de théories basées sur un comportement optimisateur et, paradoxalement, les partisans d'une telle approche ont explicitement utilisé des modèles économiques. L'étude du comportement fouilleur de Stephens et Krebs ${ }^{15}$ en constitue un excellent exemple. Ils entreprennent dexpliquer comment le comportement fouilleur de certains animaux est optimal par rapport à une certaine fonction objectif telle que le gain en énergie moyen. Ils prennent comme donnée la morphologie physique de l'animal et regardent les différents choix de la fonction objectif, encore que ceux-ci doivent être probablement considérés comme des proxies de la capacité à se reproduire. De manière caractéristique, ils montrent que certaines conditions marginales, ou de premier ordre, doivent être satisfaites par le comportement des animaux fouilleurs et déclarent que, dans de nombreux cas empiriques, c'est effectivement le cas. L'étape logique par laquelle ces auteurs complètent leur argument est, pour le lecteur non biologiste, mystérieuse. L'argument semble être le suivant. Étant donné que, avec la morphologie dont ils sont génétiquement pourvus, ces animaux optimisent une fonction objectif, ladaptation doit avoir sélectionné cette morphologie. Cette conclusion ne me semble pas logique. Les animaux n'auraient-ils pas pu simplement développer une morphologie différente et mieux adaptée à l'accomplissement de la tâche en question? L'optimisation de quelque chose par un organisme n'implique pas, selon moi, que la morphologie de l'organisme ait été sélectionnée pour le faire. Ainsi, Gould remarquait, après avoir entendu un long discours sur l'adaptation optimale du tournesol, qu'il aurait mieux valu que le

14. S. Winter, * Economic "Natural Selection" and the Theory of the Firm s, Fale Economic Essays, vol. 4, 1962, p. 225-272.

15. D. W. Stephens et J. R. Krebs, Foraging Theory, Princeton, Princeton University Press, 1986. 
tournesol développât des pieds, lesquels lui auraient aussi permis de sortir de l'ombre des hauts arbres.

Quels que soient les mérites de l'approche optimisatrice en biologie, il est intéressant de noter que deux de ses principaux partisans, Stephens et Krebs, déclarent que les biologistes évolutionnistes, comme beaucoup d'économistes, souffrent de “ jalousie pour la physique ». Ils écrivent : « De nombreux biologistes évolutionnistes veulent que leur sujet ressemble davantage à la physique classique: ils désirent ardemment sa précision, sa légitimité, sa cohérence interne, sa généralité et son double pouvoir d'explication et de prédiction ${ }^{16}$."

Ainsi, l'opposition que les économistes voient souvent entre une approche biologique et une approche physique n'est pas aussi claire qu'il semblerait.

Quelle que soit la motivation à l'origine de leur intérêt pour ce sujet, les économistes ont du mal à s'éloigner des notions d'optimisation. En effet, quand ils adoptent une optique évolutionniste, beaucoup d'économistes posent la question fondamentale suivante : la sélection naturelle conduit-elle à l'optimisation? En particulier, qu'est-ce que l'on est en train d'optimiser?'

\section{Aptitude}

Toute tentative de réponse à la première question souligne une des difficultés majeures que comporte l'utilisation de l'analogie évolutionniste en économie. À savoir, qu'entend-on précisément par aptitude? Dans la littérature biologique, on considère que l'aptitude est la capacité d'un gène à se reproduire. Dans cette même littérature, on risque toujours de tomber sur des formulations tautologiques. Cette tautologie est rendue explicite dans la façon dont on utilise les arguments évolutionnistes en théorie des jeux. Les stratégies que les dynamiques de réplication reproduisent le mieux sont celles qui rapportent le gain le plus élevé dans la matrice de jeu. Le problème est que, en général, les gains de cette matrice sont spécifiés en termes d'utilité individuelle, car autrement il n'y aurait aucun sens à calculer des espérances. En conséquence, l'aptitude semble dépendre de l'utilité de l'individu. La seule façon de réconcilier ces deux points serait de supposer que l'ensemble de la population a des fonctions d'utilité identiques, et donc que l'aptitude est correctement mesurée par les paiements. Dans cette connexion, on voit clairement pourquoi l'aspect production de l'économie a été le centre d'intérêt des économistes évolutionnistes. Ici, la fonction objectif, qui consiste simplement à maximiser le profit, est considérée sans ambiguïté comme identique entre les firmes. Il semble donc y avoir un critère constant pour évaluer l'aptitude. Une fois encore, même ici la 
question n'est, en réalité, pas si claire. Radner a montré que les firmes maximiseraient le profit espéré si elles concentraient leurs ressources sur les activités qui se sont avérées prospères. Cependant, si elles souhaitaient maximiser leur probabilité de survie, elles concentreraient leurs ressources sur les activités au rendement médiocre. En général, il ne semble pas nécessaire que ce que les individus cherchent consciemment à maximiser en termes de paiement du jeu coïncide avec l'aptitude à la reproduction. Il existe, bien sûr, une littérature substantielle au sujet de la définition de la notion d'aptitude. Mais il y a de nombreux problèmes. Certains caractères génétiques pourraient à la fois encourager une reproduction abondante et avoir des effets négatifs. Par exemple, un caractère qui produirait des affections intervenant au moment de la vieillesse n'interférerait pas avec l'aptitude à la reproduction. Je ne vais pas ici entrer dans ce débat, mais il vaut la peine de souligner que, sans une considération consciencieuse de la coïncidence entre ce qui est maximisé consciemment et ce qui assure la reproduction maximale, l'analogie évolutionniste peut être plutôt trompeuse.

Notons, de plus, que l'environnement a un sens particulier en économie. En essayant de réexaminer la théorie économique, nous perdons parfois de vue un des aspects les plus importants de l'économie, à savoir le système des prix. La désaffection vis-à-vis du modèle d'équilibre général, dont l'unique mécanisme de coordination est celui des prix, conduit souvent les auteurs à ignorer entièrement ce mécanisme. Jusqu'ici, c'est le système de prix qui détermine la profitabilité et donc, par exemple, la capacité de survie des firmes. La réaction des adaptations sur le comportement des variations de prix souligne le problème que pose un traitement approprié de l'aptitude dans les modèles en économie. Si le système de prix s'ajuste rapidement, la nature graduelle de l'adaptation peut l'empêcher d'améliorer l'aptitude dans le sens de la survie. S'il ne le fait pas, alors nous devons expliquer pourquoi il en est ainsi.

\section{Optimisation versus adaptation}

Après la discussion précédente, il est évident que, même avec un concept d'aptitude bien défini, on doit distinguer dans les comportements qui sélectionnent, et donc améliorent, celui qui adapte dans le but d'améliorer et celui qui optimise depuis le début. Le dernier constitue une hypothèse standard en théorie de l'équilibre. Le premier est le plus proche de l'idée biologique d'origine et le second est celui qui est utilisé pour justifier les équilibres. Prenons, par exemple, les jeux répétés. Dans ceux-ci, il est clair que les paiements futurs d'une action peuvent varier en fonction du choix des actions des autres agents. La situation est même plus compliquée dans les modèles économiques en général, les actions courantes influençant les revenus futurs et, en particulier, le paiement des actions futures. En d'autres termes, même si toutes les 
actions prises par les acteurs sont à la période $t$ comme elles étaient en $t-k$, les paiements peuvent être différents selon les actions prises aux autres périodes. Quand les agents tiennent compte des conséquences de leurs propres actions et de celles des autres agents sur les paiements courants et futurs, la situation devient hautement complexe. Il y a deux façons de traiter ce problème. On peut soit essayer de résoudre le problème de l'équilibre du système total $a b$ initio, soit se demander si les joueurs peuvent apprendre ou s'ajuster d'une période à l'autre, ou, pour utiliser la terminologie biologique, si un meilleur comportement peut être sélectionné et si un tel comportement peut converger vers un résultat spécifique. On peut alors comparer le point limite avec un équilibre qui apparaitrait si tous les acteurs avaient résolu le problème depuis le début. Il me semble que l'idée que les individus, de façon relativement simple, tirent les leçons de leur propre expérience et de celle des autres est plus convaincante que l'idée concurrente selon laquelle ils résolvent des problèmes de maximisation hautement compliqués, comportant des calculs de leur propre action en cours et de celle des autres. Ceci n'implique pas, bien sûr, que l'on espère nécessairement une réponse affirmative aux questions précédentes.

En poursuivant cette série d'arguments, on doit prendre une décision au sujet de l'information sur laquelle les individus basent leurs choix. Donc, même à supposer qu'ils pourraient avoir la chance d'interagir avec tous les autres, les agents individuels n'apprendront pas nécessairement quelque chose de l'expérience de tous les autres agents mais pourront, par exemple, apprendre quelque chose de l'expérience d'un sous-ensemble limité, choisi aléatoirement. Les individus pourraient alors interagir avec quelques partenaires choisis aléatoirement et réagir aux résultats de cette seule interaction. J'ai déjà mentionné une autre façon de voir ce problème, qui est de faire une simplification radicale du modèle de jeu répété général très complexe, et de priver les joueurs de toute rationalité. Ceci peut être fait en identifiant chaque agent à une stratégie fixée et en permettant alors à la sélection darwinienne d'opérer, en faisant mourir les stratégies qui réussissent le moins bien et en permettant la naissance de nouveaux agents avec les stratégies qui réussissent le mieux. C'est l’approche qui est adoptée en général dans la théorie des jeux évolutionnistes. Ceci fournit un contrepoids intéressant à la situation entièrement rationnelle, et la question qui apparaît immédiatement est de savoir si un tel type mécanique de sélection peut conduire à une situation qui aurait été atteinte par l'approche éducative. De plus, une fois que l'on prend en compte le fait que les individus interagissent seulement avec une collection limitée d'autres agents, l'évolution du système devient encore plus compliquée. Même dans le cas simple, où tous les agents peuvent interagir avec tous les autres, 
des problèmes peuvent survenir. Banerjee et Weibul1 ${ }^{17}$ considèrent une population d'individus dont certains, raisonnables, choisissent les meilleures réponses et d'autres, "programmés", ne peuvent modifier leurs choix. Ils montrent qu'après une interaction répétée, la rationalité n'est pas sélectionnée et, en outre, que des situations limites dans lesquelles des agents irrationnels survivent ne sont pas plus mauvaises du point de vue du bien-être que ces situations dans lesquelles il n'y a pas de survivants irrationnels. Bjornerstedt et al. ${ }^{18}$ démontrent que des stratégies strictement dominées peuvent survivre dans des dynamiques de sélection évolutionnistes en temps continu, certaines et raisonnables. Blum et Easley ${ }^{19}$ montrent aussi que le lien entre aptitude et rationalité est faible. Il apparaît donc évident que des caractéristiques qui semblent être meilleures d'un point de vue naturel ne sont pas nécessairement sélectionnées par ce qui semble être des dynamiques évolutionnistes plausibles. Ceci, en retour, semble ébranler l'idée que des agents apprennent rapidement à optimiser.

\section{Gènes, reproduction et spéciation}

Un des problèmes auquel on fait face en adoptant une terminologie biologique dans un contexte économique est d'établir le niveau du discours. Les gènes ont été identifiés, selon les écrits, tantôt à des stratégies, tantôt à des firmes, des routines ou des préférences individuelles. Si l'on veut parler de mutations et utiliser ce que l'on appelle des algorithmes génétiques, alors ce que l'on entend par gène doit être très clair. Le gène a été décrit à différents moments comme une routine, pour une firme considérée comme l'organisme. D'autres ont pensé la firme elle-même comme un gène. Certains auteurs souhaitent considérer la firme comme l'espèce. Dans les jeux évolutionnistes, les stratégies jouent le rôle de gène, mais la notion d'espèce n'est pas clairement définie. En effet, dans les applications économiques, le génotype est souvent mal spécifié et on l'utilise sans trop de rigueur plutôt que de le considérer comme l'élément analytique central de la théorie. Pourtant, si nous voulons prendre au sérieux l'utilisation des idées évolutionnistes en économie, ces distinctions prennent de l'importance. Considérons le problème de la reproduction. L'information génétique peut seulement être transmise à travers l'interaction des membres d'une même espèce. Comment ceci doit-il être interprété dans les modèles économiques? Jusqu'ici,

17. A. Banerjee et J. Weibull, "Evolutionary Selection and Rational Behavior „ dans A. Kirman et M. Salmon (dir.), Learning and Rationality in Econonics, Oxford, Blackwell Publishers, 1995.

18. J. Bjornestedter, M. Dufwenberg, P. Norman et J. Weibull, a Evolutionary Selection Dynamics and Irrational Survivors * dans W. Alberet et al. (dir.), Understanding Strategic Interaction. Essays in Honour of Reinhard Selten, Berlin, Springer-Verlag, 1997.

19. L. Blume et D. Easley, " Evolution and Rationality in Competitive Markets » dans A. Kirman et M. Salmon (dir.), Learning and Rationality in Economics. 
pour que l'approche évolutionniste ait une quelconque force, la notion de reproduction, qui est le moyen par lequel l'objet identifié par le gène se reproduit lui-même ou comment l'information qu'il contient est transmise, doit être bien définie.

Comme Dawkins ${ }^{20}$ le formule clairement, c'est la reproduction sexuelle qui maintient la variété au sein de l'espèce en permettant les recombinaisons. Cet aspect se perd dans la plupart des modèles biologico-économiques. Les stratégies dans les jeux évolutionnistes sont reproduites sans aucune fertilisation croisée. Il n'y a pas de recombinaison, les seuls changements qui interviennent se font par le biais des mutations. Cependant, ces dernières sont habituellement interprétées comme des transferts vers d'autres stratégies existantes. Donc, la plupart des mutations introduites dans les versions stochastiques des jeux évolutionnistes impliquent la réintroduction des éléments du pool génétique d'origine, mais n’impliquent pas l'introduction de nouvelles stratégies (voir par exemple, Foster et Young ${ }^{21}$, Kandori, Mailath et Rob' ${ }^{22}$ ainsi que Young ${ }^{23}$ ). Une fois encore, les économistes ne sont pas les seuls dans ce cas. Un certain nombre d'économistes tels que Maynard-Smith ${ }^{24}$, quand ils travaillent avec le concept de stratégie stable évolutionniste, permettent seulement lintroduction, ou l'invasion possible par des stratégies, d'un ensemble précédemment défini. Une fois encore, le modèle est donc en un certain sens fermé par le fait que l'ensemble des stratégies est fixé. Ceci, pour beaucoup, est déjà en contradiction avec l'idée de base de la biologie évolutionniste. Le nombre de branches d'un arbre est fixé et fini, et la seule question qui se pose est de savoir à quelle branche on finit. Dans le cadre d'analyse dont je viens de discuter, les notions d'espèce et de formation de l'espèce sont sans importance. Cependant, on pourrait bien se demander si c'est vraiment le cas. Il pourrait bien y avoir des limites à la façon dont les firmes peuvent créer de nouvelles stratégies à partir des vieilles. La difficulté d'injecter des pratiques américaines dans des firmes japonaises, et vice versa, pourrait nous induire à suggérer que les deux appartiennent à des espèces différentes. Pourtant, il est clair aussi que des pratiques peuvent être transférées entre des entités économiques largement différentes, uniquement par apprentissage. Dans ce cas, la notion mécanique de capacité à se reproduire devient obscure. La notion équivalente qui est habituellement invoquée pour justifier la spéciation pourrait, je suppose, être reflétée dans une sorte

20. R. Dawkins, River out of Eden, London, Weidenfeld \& Nicolson, 1995.

21. D. Foster et P. Young, "Stochastic Evolutionary Game Dynamics ", Theor. Pop. Biol. 38, 1990, p. 219-232.

22. M. Kandori, G. J. Mailath et R. Rob, "Learning, Mutation, and Long Run Equilibria in Games n, Econometrica, vol. 61, $\mathrm{n}^{\circ}$ 1, janvier 1993, p. 29-56.

23. H. P. Young, "The Evolution of Conventions ", Econometrica, vol. 61, $\mathrm{n}^{0} 1$, janvier 1993, p. 57-84.

24. J. Maynard-Smith, Evolution and the Theory of Ganes, Cambridge University Press, 1982. 
de séparation culturelle, mais ceci n'est rien de plus qu'une spéculation hasardeuse.

Il est clair que certains des choix que jai décrits, particulièrement ceux qui concernent les mutations, ne sont pas innocents. En effet, et je reviendrai sur ce point, dans de nombreuses utilisations de l'analogie évolutionniste, les théoriciens de l'économie ont tenté d'utiliser l'évolution comme un moyen de sélectionner entre des stratégies existantes, plutôt que comme un moyen de dépeindre un monde illimité en développement. En effet, Samuelson ${ }^{25}$ développe entièrement ce thème autour de l'idée de sélection des équilibres. Bien que la commodité de cette approche soit évidente, on est quelque peu tiré en arrière vers le monde de la théorie économique standard, dans lequel on cherche la convergence ou la stabilité d'un équilibre particulier.

\section{La theorie des jeux évolutionnistes}

Certains accepteront difficilement que la théorie des jeux puisse être classée sous l'enseigne de l'économie néoclassique. Pour ceux qui identifient cette dernière à une approche méthodologique individualiste, l'association paraîtra désagréable puisque le comportement stratégique est conceptuellement très différent de la concurrence pure. Cependant, ceux qui l'identifient avec cette idée que les individus maximisent sous contrainte, trouveront eux aussi déplaisante cette conception de la théorie des jeux. Ceci étant dit, on doit aussi admettre que dans les modèles de jeux évolutionnistes les plus standards, les individus ne maximisent rien. Néanmoins, la théorie des jeux évolutionnistes fournit une très bonne base pour examiner l'analogie biologique puisque les termes y sont consciencieusement définis et l'analyse, le plus souvent rigoureuse.

Le modèle de base comporte un jeu sous-jacent avec un ensemble de stratégies qui est habituellement indépendant du temps et fini. Les individus y sont identifiés avec les stratégies. En tout point, l'état du système sera défini par les proportions des différentes stratégies présentes dans la population. On peut considérer soit que les individus ont un succès, mesuré par leurs paiements espérés, par rapport à la population courante, soit que les stratégies sont tirées par deux, au hasard, dans la population courante. Le succès d'une stratégie particulière dans une large population sera alors le même que la valeur espérée de chaque individu, identifié avec sa stratégie. Une première étape est d'examiner les situations qui sont en un sens stables. Le critère utilisé ici correspond typiquement à la stabilité évolutionniste, ce qui signifie simplement que la combinaison stratégique existante n'est pas vulnérable face à une petite invasion d'une autre stratégie. On fait souvent référence à cette dernière 
comme mutation, une notion qui a connu un succès certain en biologie depuis les travaux innovateurs de Maynard-Smith. Cependant, nous nous intéressons ici à la robustesse de l'équilibre. Quelles sont les dynamiques du processus?

Imaginons maintenant que le jeu est en train de se dérouler, et supposons que certains individus meurent et sont remplacés à chaque étape. Un critère de sélection détermine à quelle vitesse les stratégies vont se développer comme une fonction de leur succès. Puisque ce dernier est mesuré par rapport aux paiements reçus par les stratégies, toute distinction entre ce qui est maximisé consciemment et ce qui constitue une aptitude disparaît.

Il serait inutile de donner ici un compte rendu des résultats qui ont été obtenus en analysant les dynamicues dites "de réplication ", dynamiques que l'on utilise habituellement pour s'attaquer à ce genre de problème. Dans ce cas, on analyse le processus évolutionniste comme un processus déterministe et souvent, on en fait de plus un processus en temps continu. Hofbauer et Sigmund ${ }^{26}$ constituent la référence classique en la matière et Weibull donne un traitement complet de cette approche. Il discute en détail les dynamiques de tels processus. Le problème revient à analyser les propriétés d'un système d'équations différentielles ordinaires. Le résultat de base est que, à long terme, seules survivront les stratégies non strictement dominées de façon itérative. En particulier, si nous considérons les jeux doublement symétriques, dans lesquels la matrice des paiements à laquelle tous les joueurs font face est la même, alors les stratégies stables de façon asymptotique des dynamiques de réplication sont les mêmes que les stratégies stables au sens évolutionniste du jeu sousjacent. De plus, l'aptitude moyenne de la population augmente au cours du temps.

Tout cela, me semble-t-il, pourrait tirer la sonnette d'alarme pour ceux qui pensent que l'économie change de façon évolutionniste. Dans tous les cas, il semble que l'on se soit passé de plusieur's caractéristiques de l'évolution. Le tâtonnement stochastique graduel et aveugle du processus évolutionniste a cédé la place à quelque chose de beaucoup plus mécanique et déterministe. À cette étape du raisonnement, deux questions valent la peine d'être posées. L'approximation du système stochastique d'appariement aléatoire par un système déterministe est-elle inoffensive? Que se passe-t-il si les mutations sont très fréquentes? La réponse à la première question est donnée par Boylan. Il analyse un jeu simple dans lequel deux agents sont appariés et produisent deux descendants. Il montre que les résultats peuvent être très différents selon la façon dont on pose les limites pour obtenir le système déterministe continu. Ceci

26. J. Hofbauer et K. Sigmund, The Theory of Evolution and Dynanical Systens, New York, Cambridge University Press, 1988. 
constitue une mise en garde contre l'utilisation d'une approche déterministe pour analyser l'évolution.

La réponse à la deuxième question soulève de nouveaux problèmes. Que sont les mutations dans ce contexte :' $\lambda$ quelle fréquence apparaissent-elles? Dans l'optique de Binmore el Samuelson, on peut imaginer un processus dajustement de hase, perturbé de temps en temps par une mulation, que lon intcrprète comme un changement aléatoire de stratégies. Dans ce cas, nous pouvons maintenant chercher une distribution stationnuire qui décrira les proportions de temps que le système passera dans chaque élat. On entend ici, par état, le vecteur des parts de chaque stralégic. Si, dans un jeu de coordination simple, il y a accord sur une solution, dans un contexte déterministe le système ne s'en écartera donc pas. Cependant, si l'on rajoute des mutations aléatoires, le systéme sorientera tôt ou tard vers une autre solution. Le délai peul être très long. mais cela finira par arriver. Aussi Binmore et Samuclson considèrent-ils comme pertinent pour cetle distribution stationnaire l'expression ultra-long terme. Fondamentalement, l’idée est que, à long terme, le système peut se fixer en un élat mais, à ultua-long terme, il peut toujours changer d'un état à lautre. Une lelle interprétation des mutations noest pas nécessaire. Elles pourraient consister en expérimentations. Si les individus, par exemple, ont la possibilité, en faisant des expériences, de diriger le système vers un état supérieur, ils peuvent essayer occasionnellement de le fairc. Do façon alternative, les joueurs peuvent simplement faire dles erreurs de temps en temps. Il est difficile de réconcilier cette derniere interprétation avec l’idée que les individus sont liés à certaines stratégies, mais on pourrait à la place considérer que les acteurs sont remplacés de temps en temps par de nouveaux acdeurs.

La question importante ici est de savoir si les mulations changent les caractéristiques de très long terme du système. Kandori, Mailath et Rob ${ }^{27}$ ainsi que Young semblent montrer de façon convaincante que cest le cas dans des modèles où les agents choisissent leuts stratégies. Ici, pour rester proches des modèles inspirés par la biologie, supposons que des individus peuvent changer de stratégies de temps en temps, mais choisissent dans ce cas la meilleure réponse dans la situation courante. Clairement, si le système est proche d'un équilibre de Nash strict, la distribution des stratégies convergera el ne changera plus en l'absence de mutations. Un tel équilibre pourait donc être un point fixe du processus. En outre, le système pourrait cycler pour d'autres processus évolutionnistes. Kandori, Mailath et Rob ainsi que Young montrent néanmoins que l'introduction de mutations change radicalement la situation. L'idée est d'introduire une petite probabilité $\varepsilon$ qu'un agent choisira une stratégie qui n'est

27. M. Kandori, G. J. Mailath et R. Rob, "Learning, Mutation, and Long Run Equilibria in Games.

28. H.P. Young, "The Evolution of Conventions ». 
pas la meilleure. Ils laissent ensuite $\varepsilon$ tendre vers zéro et montrent que la distribution stationnaire limite affectera un poids zéro à certains équilibres. Ils sélectionnent donc certains équilibres. Cela paraît encourageant puisque cela suggère que, à très long terme, le système passera essentiellement tout son temps dans les états d'équilibre qui ont été sélectionnés. L'intuition est claire. Si le système a besoin d'une poussée de mutations pour sortir d'un équilibre A, mais d'une bien plus grosse poussée pour sortir de B, alors quand les mutations deviennent de moins en moins fréquentes, la probabilité d'être en A tend vers zéro. Cependant, ceci a récemment été contesté par Bergin et Lipman ${ }^{29}$. Ils suggèrent que le défaut de l'argument précédent est que l'on ne permet pas à la fréquence des mutations de dépendre du temps. Si certains états sont "meilleurs" que d'autres, n'y a-t-il pas moins d'expérimentations à partir de ceux-ci ? Prenons le jeu de coordination simple suivant:

A $\quad$ B
A $\quad(2,2)$
$(0,0)$
B $\quad(0,0)$

L'état du système peut être caractérisé par le nombre des $\mathrm{N}$ joueurs jouant $A$. Il est clair que si moins d'un tiers des joueurs jouent $A$ et que tous les joueurs peuvent choisir en chaque point, alor's, en l'absence de mutations, le système sautera à l'état 0 et y restera. Il sautera à l'état $\mathrm{N}$ à partir de tout état au-delà de $\mathrm{N} / 3$. Donc $\mathrm{P}$, la matrice de transition $\mathrm{N} \times \mathrm{N}$ de ce problème, aura une forme très particulière. Cependant, nous pouvons à présent ajouter des $\varepsilon$ pour rendre $P$ strictement positive et la matrice $M$ résultante aura une distribution stationnaire unique: Maintenant, adoptons l'astuce qui consiste à laisser les $\varepsilon$ tendre vers zéro à un rythme qui dépend de l'état dans lequel le système se trouve, alors la distribution stationnaire limite changera. Bergin et Lipman montrent qu'en choisissant les $\varepsilon$ de façon appropriée, toute distribution limite stationnaire, qui est une limite d'une séquence de distribution stationnaire de matrices dérivées de $P$ et distribuées positivement, peut être atteinte. Tout événement de poids un sur A et de poids un sur B peut donc arriver. Sans une quelconque justification a priori du choix des perturbations ou mutations, aucune sélection ne peut être effectuée.

Cependant, mon propos est de traiter de cette application particulière de la théorie évolutionniste. Quand on utilise le terme de

29. J. Bergin et B. L. Lipman, "Evolution with State-Dependent Mutations ", Econometrica, vol. 64, 1996, p. 943-956. 
mutation sans prêter une attention particulière à son interprétation, le rythme auquel elles arrivent peut conduire à des résultats clairs, mais pas nécessairement très éclairants. Pour revenir sur ce point, j'aimerais mentionner un autre exemple, dans lequel la structure sous-jacente du modèle est très importante pour la détermination du développement du système. Il est issu d'Ellison ${ }^{30}$. Ce dernier considère en premier lieu un modèle avec une interaction globale et le compare ensuite à un autre modèle, dans lequel les joueurs sont situés sur un cercle. Dans chaque cas, ils jouent le jeu de coordination simple décrit ci-dessus el qui comporte deux équilibres, l'un dominant l'autre. Dans le cas global, ils jouent contre un membre choisi dans la population lotale ce, dans le cas local, contre un de leurs voisins immédiats. Dans le contexte évolutionniste, l'aptitude dépend, dans le dernier cas, de l'environnement local, c'està-dire des stratégies jouées par les voisins. Dans ce modèle, comme je l'ai indiqué, la bonne stratégie $A$ constitue le meilleur choix si au moins un tiers des partenaires jouent $\Lambda$, puisque le paiement est deux lois ce qui est obtenu quand les jouems se coopdonnent sur la manvaise stratégie. Donc, daus le cas local, on choisira toujours la bonne stratégie si au moins un des deux voisins a choisi cette stratégie, alor's que dans le modèle avec une interaction globale, on a besoin qu'un liers de tous les joueurs joment la bonne stratégie pour avoir intérồ à la choisir. À présent, il y a deux bassins d'attraction du processus dynamique par lequel les agents modifient leurs stratégies : celui dans lequel les agents choisissent tous la mauvaise stratégic el celui dans lequel ils choisissent tous la bonne. Si nous supposons encore que, de temps en temps, un agent change sa stratégie de façon stochastique, c’est-à-dire qu'il y a des mutations aléaloires, de nombreuses mulations de co type seront nécessaires, dans le cas global, avant que le système séearle du mouvenis ou du bon. bassin d'athaction. Cependant, el cest peul-ête étonnant, dans le systeme d’interachon locale, la bomme stralégie pent émerger de la poprulation aussitôt quin joucur ou, scelon le nombre d"individus lans le systeme, deux joucents adjacents choisissent eotle stratégie: sans un queleongue ehangement aléatoire supplémentaine. l, interaction locale facilite donc des ehangements plus rapides vers une coordination agrégée sur un meilleur comportement. Là encore, le ehoix de la suruchure du modele joue un rôle erucial dans la détermination des dynamiques du systeme et il n'y a pas d'indication claire au sujet du type particulier d"équilibre qui sera privilégié par les dynamiques évolutionnistes. Bien que j'aie une préférence pour l'approche stochastique, qui me semble être davantage dans l'esprit. d'origine des modèles évolutionnistes, on ne peut encore lirer aucune conclusion nelte au sujet de son utilité.

30. G. Ellison, "Learning, Local Interaction and Co-ordination ", Econometrica, vol. 61 , septembre 1993, p. 1047-1072. 
Cette brève revue de la place des idées évolutionnistes dans la théorie économique a omis un certain nombre de caractéristiques importantes de l'économic évolutionniste. Ceci élant paree que ces caractéristiques concernent des points que l'économie dominante ne parvient pas à expliquer et auxquels elle ne se rélère donc pas. Par exemple, à quelle fréquence les mutations arrivent-elles?' Y a-t-il des groupes subits de mutations qui changent entièrement l'équilibre du systène? Celte idée d'unc évolution par sauts (sallalion), associéce au nom de Golschmidt, se traduit en économic par la question: pourquoi semble-t-il y avoir parfois des poussces d'innovalion, par exemple? Un tel phénomòne semail compatible avec des innovations conçues eomme le résultal d"un flot continu de changements aléatoires, mais oceasionnellement miles. De temps en temps, de tels événements uliles s’aceumulemicnt inćvilablement, produisant une breve périote d'inslabilicé. (3) encore, il pent parfois arriver qu'un petil changement au niveau génétique produise un changement dramatique au niveau des phénotypes. Le phénomène du changement technologique est l'un des plus mal traités par la thécie. standard qui, habituellement, pose comme principe l'arrivée d'un flot de chocs exogènes agrégés sur l'économie. La théorie de la croissance endogène ne permet pas non plus d'expliquer, sauf par analogic, l'arrivée du progrès technologique qui relance la croissance.

Cependant, mon examen plutôt superficiel montre deux choses concernant l'influence de la pensée évolutionniste sur l'économie dominante. Dautre part, bien que l'analogie ail été largement utilisée, son véritable sujet a été en général de décrire comment un système pourrait, par un processus d'apprentissage, se diriger vers un équilibre. Les équilibres en question sont bien définis dès le départ et, après un apprentissage déterministe ou aléaloire, le système se: trouve dans l'un d'entre eux. Les mutations sont considérées comme des changements dans les stratégies ou les actions et la plus apte d'entre elles est sélectionnéc. Celle approche nous oblige à replacer une sorte d'agent de coordination central, du type du commissairepriseur walrasien. D'autre part, comme Samuelson ${ }^{31}$ le suggère, l'étude des jeux évolutionnistes peut évoluex de la môme façon que l'étude des processus d'ajustement dans l'économie waltasienne. Il se peut qu'elle s'amenuise et laisse finalement inchangée la conception que les économistes ont des équilibres. Dans le cas walrasien, les résultats de Sonnenschein, Mantel et Debreu rendent ceci presque inévitable, mais ils ne résolvent pas les objections logiques à la théorie de l'équilibre statique. Des résultats récents risquent de produire des réactions similaires envers la théorie des jeux.

31. L. Samuelson, Evolutionary Games and Equilibrium Selection. 
Mon impression est qu'il y a une incompatibilité fondamentale entre une vision de l'économie considérée comme un système en développement illimité, dans lequel l'environnement et la nature des acteurs sont sujets au changement, que je considère comme proche de la situation biologique, et une vision de l'économie considérée comme un système fermé avec un ensemble fixe d'états d'équilibre. Si nous adoptons la dernière approche, le seul moyen pour que l'économie puisse acquérir des propriétés dynamiques est de la soumettre à des chocs agrégés, arbitraires el non définis. Bien que ce soit ce qui est effectivement fait en macroéconomie, cela vaut sûrement la peine de poursuivre l'approche plus ambitieuse, dans laquelle l'analyse évolutionniste est plus quiune vague analogie.

\section{EHESS}

Université d'Aix-Marseille III 